\title{
Target Exploration for Disconnected Feasible Regions in Enterprise-Driven Multilevel Product Design
}

\author{
Harrison M. Kim* \\ University of Illinois at Urbana-Champaign, Urbana, Illinois 61801 \\ Deepak K. D. Kumar ${ }^{\dagger}$ and Wei Chen \\ Northwestern University, Evanston, Illinois 60208 \\ and \\ Panos Y. Papalambros ${ }^{\S}$ \\ University of Michigan, Ann Arbor, Michigan 48109
}

\begin{abstract}
Enterprise-level business decisions are linked with engineering product decisions by integrating enterprise utility optimization and engineering design optimization under a hierarchical, multilevel, decision-based design framework. The enterprise problem sets attribute targets, that is, specifications, for engineering product development, which then optimizes product performance within the feasible design space to match the targets with minimum deviations. When the feasible domain imposed by engineering product development is disconnected in the space of attribute targets, an engineering design with the minimum deviation from the targets may not correspond to the design with the maximum utility value, even though the design is a converged solution from the multilevel optimization. To address this issue, a new algorithm is developed, which systematically explores the target space to lead the engineering product development to a feasible and optimal design in the enterprise context. Analytical examples and an automotive suspension design case study are presented to demonstrate the effectiveness of the proposed methodology.
\end{abstract}

\section{Introduction}

D ESIGNING a large-scale artifact involves multidisciplinary efforts in marketing, product development and production. There have been a number of efforts to integrate enterprise economic considerations in engineering design, for example, the efforts of Donndelinger and Cook, ${ }^{1}$ Besharati et al., ${ }^{2} \mathrm{Li}$ and Azarm, ${ }^{3}$ Wassenaar and Chen, ${ }^{4}$ Cooper et al., ${ }^{5}$ Gu et al., ${ }^{6}$ Michalek et al., ${ }^{7}$ and Markish and Willcox. ${ }^{8}$ They have demonstrated the interaction between marketing and engineering in an enterprise. In this paper, the enterprise is defined as the organization that designs and produces an artifact to maximize its utility, for example, profit. For simplicity, marketing, production planning, and other enterpriselevel activities are referred to as enterprise-level product planning; engineering-related design activities are referred to as engineering product development.

Emphasis on enterprise-driven design models has led to incorporating demand and profit models that capture both producer and consumer needs into product design. For example, Wassenaar and $\mathrm{Chen}^{4}$ developed demand models utilizing discrete choice analysis ${ }^{9}$ in a decision-based design (DBD) framework. ${ }^{4,6,10}$ The demand models capture the choices that customers make as functions of customerproduct-selection attributes, that is, product attributes that are of

Presented as Paper 2004-4546 at the AIAA/ISSMO 10th Multidisciplinary Analysis and Optimization Conference, Albany, NY, 30 August-1 September 2004; received 11 October 2004; revision received 5 August 2005; accepted for publication 9 August 2005. Copyright (C) 2005 by Wei Chen. Published by the American Institute of Aeronautics and Astronautics, Inc., with permission. Copies of this paper may be made for personal or internal use, on condition that the copier pay the $\$ 10.00$ per-copy fee to the Copyright Clearance Center, Inc., 222 Rosewood Drive, Danvers, MA 01923; include the code 0001-1452/06 \$10.00 in correspondence with the CCC.

*Assistant Professor, Department of General Engineering; hmkim@ uiuc.edu. Member AIAA.

${ }^{\dagger}$ Graduate Student, Department of Mechanical Engineering, 2145 Sheridan Road, Tech B224; k-dileep@ northwestern.edu.

$¥$ Associate Professor, Department of Mechanical Engineering, 2145 Sheridan Road, Tech B224; weichen@ northwestern.edu. Associate Fellow AIAA.

${ }^{\S}$ Professor, Department of Mechanical Engineering; pyp@umich.edu. Senior Member AIAA. interest to customers. Along with cost models, the Wassenaar et al. DBD approach ${ }^{11,12}$ has been used to obtain the optimal settings of product attributes at the enterprise level to maximize the net revenue of a firm, considering engineering needs, socioeconomic and demographic background of customers, and time. In the Wassenaar et al. approach, the focus was on identifying the optimal product attributes, rather than linking the product attributes with engineering design process. In this paper, following the DBD framework, a multilevel optimization formulation is proposed to link enterpriselevel decision making with engineering-level product design and to treat the engineering product development as a process of meeting performance targets identified from the enterprise decision making. Furthermore, a new algorithm is developed to explore the potentially disjoint performance target space systematically and to lead the engineering product development process to a feasible and optimal design for the enterprise.

Under the existing DBD framework, all product design decisions, engineering or non-engineering, are made simultaneously (all-inone) to optimize the enterprise-level design objective, that is, to maximize the expected utility, expressed as a function of net revenue (profit). This all-in-one (AIO) approach, shown in Fig. 1a, is desirable, but often practically infeasible due to the computational and organizational complexity. From the viewpoint of organizational infrastructure of a company, decomposition and multilevel distributed approaches must be introduced. Figure $1 \mathrm{~b}$ shows a decomposed view of the interaction between enterprise-level product planning and engineering product development in a hierarchical, multilevel optimization framework. The presented fraremwork shares similar ideas to those of Cooper et al. ${ }^{5}$ and Michalek et al. ${ }^{7}$ that treat engineering design as a hierarchical process of meeting design targets at different levels. The difference is that this work follows the DBD practice in formulating the enterprise-level optimization problem and uses the discrete choice analysis techniques for demand modeling. As detailed in Sec. II, the demand model provides a link between engineering product development and enterprise planning. Following Fig. 1b, the enterprise-level product planning problem maximizes the utility $V$ with respect to the target performance $\boldsymbol{T}^{U}$ and the rest of the enterprise decision variables $\boldsymbol{x}_{\text {ent }}$, subject to enterprise-level design capability $\boldsymbol{g}_{\mathrm{ent}} \leq \mathbf{0}$ and $\boldsymbol{h}_{\mathrm{ent}}=\mathbf{0}$. When the utility is maximized, the corresponding target performance is defined as the utopia target. 


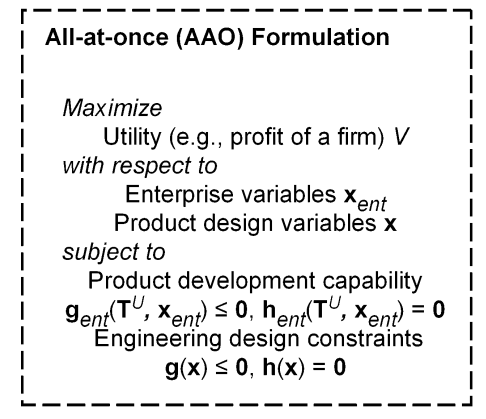

a) Original AIO formulation

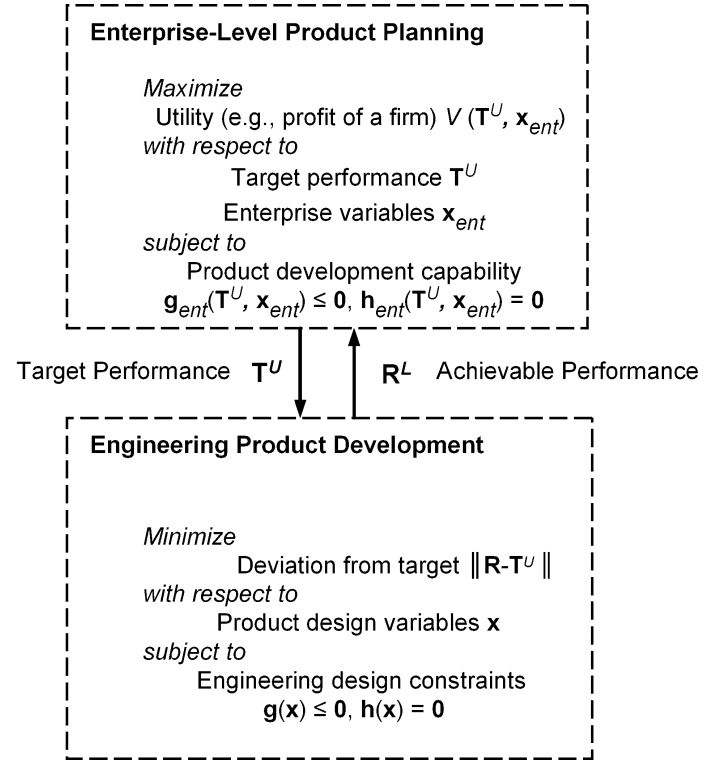

b) Decomposed formulation

Fig. 1 Interaction between enterprise product planning and engineering product development.

Following current industrial practice, we view the engineering product development process as a process where targets set at the enterprise level are met through product realization. At the engineering level, the objective is to minimize the deviation between the performance target and the achievable product performance response $\boldsymbol{R}$ while satisfying the engineering feasibility constraints $\boldsymbol{g}$ and $\boldsymbol{h}$.

When the integrated enterprise and engineering problem is solved using multilevel optimization, it is essential to ensure preference consistency: Smaller deviation from the targets in engineering product development corresponds to a higher enterprise-level utility, so that the solution from the multilevel optimization procedure (a decomposed problem, Fig. 1b matches the AIO solution of the integrated enterprise and engineering problem (Fig. 1a). Ensuring preference consistency is not very straightforward with a decomposed formulation. Under a multilevel design framework, an ideal product development scenario is when the targets corresponding to the best enterprise utility, that is, the utopia target, would lead to an engineering design matching the targets perfectly. A perfect match may be rare due to engineering constraints introduced at the product development level. Additionally, as further detailed in Sec. III, if the feasible domain imposed by the engineering product development is disconnected, that is, disjoint, in the space of attribute targets (engineering performance domain), the engineering design with the minimum deviation from the targets may not correspond to the design with the maximum utility value, even though the design is a converged solution from the multilevel optimization. Disconnected feasible performance domains often occur in complex systems design when there are multiple engineering disciplines involved in engineering development and when each discipline can seek distinctly different design alternatives in engineering development. In the vehicle suspension design case study of Sec. IV, a vehicle manufacturer maximizes the enterprise utility based on two disconnected feasible target performance domains imposed by suppliers of suspension components. The suppliers (or engineering design teams) try to achieve the utopia targets (suspension stiffnesses) as closely as possible, but it is assumed that a perfect match is not possible.

One of the main contributions of this work is an algorithm that overcomes this difficulty. The algorithm can systematically explore attribute targets in the disconnected feasible domain to lead the engineering product design process to finding a feasible and optimal design in the enterprise context. The proposed algorithm in Sec. III guides the enterprise-level decision maker to assign alternative targets so that the enterprise maximizes the net revenue and the suppliers achieve the targets as closely as possible. The adjustment of targets set at the enterprise level guides the exploration within a disconnected engineering performance feasible domain.
This adjustment may shift the enterprise utility value away from its original utopia value. In return, however, a better, that is, higher utility and feasible design can be obtained satisfying the engineering constraints.

The proposed formulation uses a bilevel hierarchical structure that does not entail competition in their interaction. The interaction nature between enterprise product planning and engineering product development is similar in concept to the leader/follower model, ${ }^{13-17}$ except that the existing applications of game theory emphasize interactions between different engineering groups, whereas our work focuses on the interaction between upper enterprise needs and lower engineering capabilities (Fig. 1b). Also, the traditional disjunctive programming literature focuses on modeling a problem with disjunctive feasible domains utilizing mixed integer nonlinear programming problem formulations, and conventional search algorithms, such as branch and bound, are applied to solve them. ${ }^{18-20}$ Considering disconnected (or disjoint) feasible space is conceptually similar to the work here, but the proposed algorithm focuses on locally "exploring" the neighboring region of the performance target, rather than globally searching for the solution in an enumerative way.

In this work, the engineering product development problem at the bottom of the hierarchy in Fig. 1 is viewed as a hierarchical process by itself. To achieve the targets identified from enterprise product planning, the analytical target cascading (ATC) approach ${ }^{21-24}$ is adopted for the hierarchical product development process. ${ }^{5,7}$ ATC is a multilevel, multidisciplinary design methodology to find an optimal system design, ensuring consistency among subsystems or disciplines and achieving the overall product targets assigned at the top of the hierarchy. In the field of multidisciplinary design optimization (MDO), several design architectures have been developed to support collaborative, multidisciplinary design environment using distributed design optimization, for example, concurrent subspace optimization, ${ }^{25}$ bilevel integrated system synthesis (BLISS), ${ }^{26}$ and collaborative optimization (CO). ${ }^{27}$ A comprehensive review of MDO architectures is provided by Sobieszczanski-Sobieski and Haftka ${ }^{28}$ and Kroo. ${ }^{29}$ Note that the engineering product development can be formulated following any of the available multilevel MDO formulations depending on either the hierarchical or nonhierarchical characteristics of decision flow.

The paper is organized as follows. The background of the enterprise product planning model based on DBD principles and the hierarchical product development model based on ATC are first provided in Sec. II. The proposed solution algorithm is presented in Sec. III. In Sec. IV, the proposed approach is demonstrated and verified using three examples: an analytical example with a single 
optimum in the utility space, an analytical example with multiple local optima in the utility space, and a vehicle suspension design example that includes the modeling of customer preference choices of vehicle systems. In Sec. V, the closure is provided.

\section{Enterprise Product Planning and Engineering Product Development Model}

In this section, some detail of the product planning model at the enterprise level and the product development model at the engineering level are provided. The enterprise-level product planning model is to maximizes a utility, such as the profit of a firm, that entails the development of demand and cost models. The engineering-level product development model is to minimizes the deviations from the targets set by the enterprise level problem, which is formulated based on the ATC approach.

As shown in Fig. 2, the demand $Q$ plays a critical role in assessing both revenue and expenditure and, ultimately, the profit, that is, net revenue $V$. To use the enterprise model to guide engineering product development, $Q$ is expressed here as a function of the customer-product-selection attributes $\boldsymbol{A}$, socioeconomic attributes $S$ of the market population, price $P$, and time $t$; the expenditure $C$, that is, life cycle cost including manufacturing cost and others, is a function of the customer-product-selection attributes $\boldsymbol{A}$, the engineering attributes $\boldsymbol{E}$, exogenous variables $\boldsymbol{y}_{\mathrm{ex}}$, demand $Q$, and time $t$. The customer-product-selection attributes $\boldsymbol{A}$ are product features (next to brand, price, and warranty) that a customer typically considers when purchasing the product, and the engineering attributes $\boldsymbol{E}$ are quantifiable product properties that can be directly optimized by design engineers, but indirectly affect purchasing behavior and manufacturing cost. For example, customer preference for ride quality of a vehicle is influenced by the stiffness of the suspension springs. Here the stiffness of spring is an example of the engineering attribute that influences demand and net revenue, but indirectly affects purchasing behavior of a customer. At the enterprise level, targets $\boldsymbol{T}$ are set for both $\boldsymbol{A}$ and $\boldsymbol{E}$. To assist the selection of design alternatives in engineering development, the relationships of $\boldsymbol{A}$ and $\boldsymbol{E}$ with design options need to be established through engineering analyses.

Demand modeling using discrete choice analysis (DCA) and conjoint analysis has been widely used in the marketing and transportation communities..$^{9,30,31}$ Researchers in the design community ${ }^{2-4,7}$ have also had success in integrating such models in their work for product design. In this paper, the DCA approach is adopted for demand modeling. The multinomial logit (MNL) model is used because it has a closed form and uses the Gumbel (see Ref. 9) error distribution that closely approximates the normal distribution, a more realistic assumption for the error distribution when estimating choice behavior. The choice probability when using the MNL model is shown in Eq. (1), where $\operatorname{Pr}_{n}(i)$ is the probability that respondent $n$ chooses alternative $i, J$ is the choice set that is available to individual $n$, and $W$ is the observable/deterministic part of the utility function of customer:

$$
\operatorname{Pr}_{n}(i)=e^{W_{i n}} / \sum_{j \in J} e^{W_{j n}}
$$

This formulation implies that the probability of choosing an alternative increases monotonically with an increase in deterministic utility of that alternative and decreases with the increase of deterministic utility of any or all of the other alternatives. Using the MNL model imposes the independence of irrelevant alternatives (IIA) property, which makes the calculation of choice probabilities much easier. There are limitations associated with the IIA property; however, the explanation of them is beyond the scope of the current work.

In this work, the engineering product development problem (lower level in Fig. 2) as a hierarchical process is solved using the ATC,${ }^{21-24}$ a multilevel optimization approach. A key difference between ATC and most of the MDO formulations, including $\mathrm{CO},{ }^{27}$ is that, with ATC, the original problem is decomposed hierarchically at multiple levels, whereas the interconnections between the multiple subsystems at each level are considered and coordinated at one level above. The ATC problem $P_{i j}$ associated with the $j$ th element at the $i$ th level of the hierarchy (Fig. 3) is formulated as

$$
\begin{aligned}
P_{i j}: & \min _{x_{i j}} \boldsymbol{w}_{i j}^{\tilde{R}}\left\|\tilde{\boldsymbol{R}}_{i j}-\boldsymbol{T}_{i j}\right\|+\boldsymbol{w}_{i j}^{R}\left\|\boldsymbol{R}_{i j}-\boldsymbol{R}_{i j}^{U}\right\| \\
& +\boldsymbol{w}_{i j}^{y}\left\|\boldsymbol{y}_{i j}-\boldsymbol{y}_{i j}^{U}\right\|+\boldsymbol{\varepsilon}_{i j}^{R}+\boldsymbol{\varepsilon}_{i j}^{\boldsymbol{y}}
\end{aligned}
$$

subject to

$\sum_{k \in C_{i j}}\left\|\boldsymbol{R}_{(i+1) k}-\boldsymbol{R}_{(i+1) k}^{L}\right\| \leq \varepsilon_{i j}^{R}$

$\sum_{k \in C_{i j}}\left\|\boldsymbol{y}_{(i+1) k}-\boldsymbol{y}_{(i+1) k}^{L}\right\| \leq \varepsilon_{i j}^{y}$

$\boldsymbol{g}_{i j}\left(\hat{\boldsymbol{R}}_{i j}, \boldsymbol{x}_{i j}, \boldsymbol{y}_{i j}\right) \leq \mathbf{0}$

$h_{i j}\left(\hat{\boldsymbol{R}}_{i j}, \boldsymbol{x}_{i j}, \boldsymbol{y}_{i j}\right)=\mathbf{0}$

Elements $j$

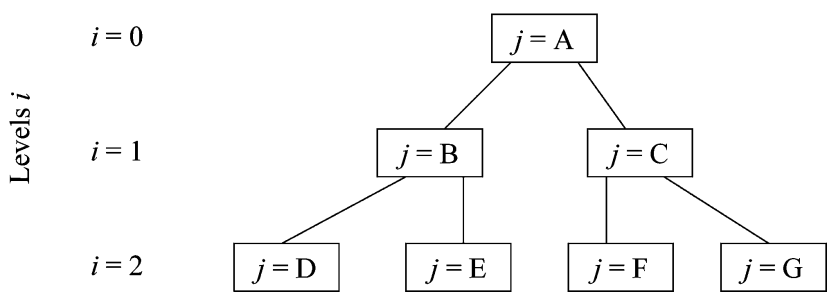

Fig. 3 Decomposition example with three-level hierarchy.
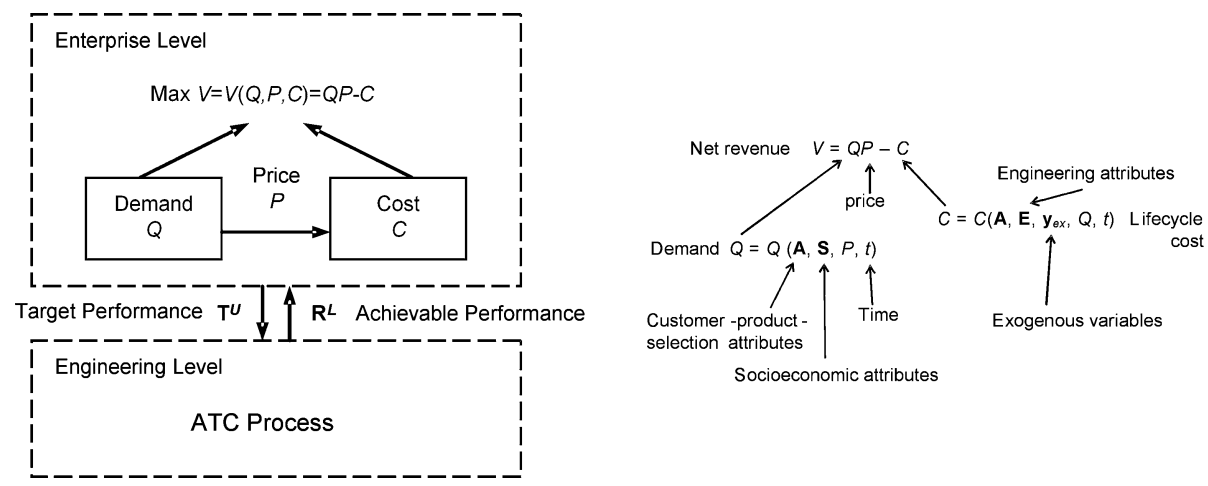

Fig. 2 Bilevel enterprise decision flow and net revenue model. 


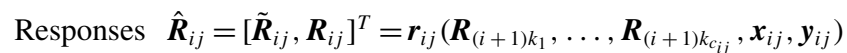
are computed by means of analysis and/or simulation models. The vector of all optimization variables is $\hat{\boldsymbol{x}}=\left[\boldsymbol{x}_{i j}, \boldsymbol{y}_{i j}\right.$, $\left.\boldsymbol{y}_{(i+1) k_{1}}, \ldots, \boldsymbol{y}_{(i+1) k_{c_{i j}}}, \boldsymbol{R}_{(i+1) k_{1}}, \ldots, \boldsymbol{y}_{(i+1) k_{c_{i j}}}, \boldsymbol{\varepsilon}_{i j}^{R}, \boldsymbol{\varepsilon}_{i j}^{y}\right]^{T}$, and $\boldsymbol{x}_{i j}$ is the vector of local design variables. $\tilde{\boldsymbol{R}}_{i j}$ corresponds to responses linked to local targets, and $\boldsymbol{R}_{i j}$ corresponds to responses linked to cascaded targets. Here $\varepsilon_{i j}^{R}$ and $\varepsilon_{i j}^{y}$ are the tolerance optimization variables for ensuring consistency. Superscripts $(\cdot)^{U}$ and $(\cdot)^{L} \mathrm{de}-$ note values passed down and up from the upper and lower levels, respectively. The vector $\boldsymbol{T}$ denotes local targets, and $\boldsymbol{g}_{i j}$ and $\boldsymbol{h}_{i j}$ are local design constraints. Weights $\boldsymbol{w}$ are assigned to the deviation terms in the objective, and the convergence behavior of the ATC process can be affected by the way weights are set (or updated). In this work, the weights are set to reflect preferences after normalizing the individual deviation terms. For this, the reader can refer to weight update schemes and their effects on convergence. ${ }^{32-34}$

Most of the other work considered up to this point in MDO research, for example, BLISS ${ }^{26}$ and $\mathrm{CO},{ }^{27}$ has been concerned with decomposing a problem into a series of problems, all at one nonhierarchical level, but then solving the problem using bilevel optimization formulations. ATC operates by formulating and solving a minimum deviation optimization problem for each element in the hierarchy. Compared to the existing bilevel optimization formulations, the multilevel hierarchical modeling facilitated by the ATC approach better matches a multilayered decision making organization, where subsystems and components can be supplied by different organizational units or outsourced to independent companies.

\section{Multilevel Enterprise and Engineering Problem Formulation and Solution Algorithm}

The original unconstrained enterprise level utility optimization problem is

$$
P_{\mathrm{ent}}^{0}: \max _{T, x_{\mathrm{ent}}} V\left(\boldsymbol{T}, \boldsymbol{x}_{\mathrm{ent}}\right)
$$

where the objective is to maximize the utility $V$ that is a function of design attribute targets $\boldsymbol{T}$ for the engineering problem and of other enterprise variables $\boldsymbol{x}_{\text {ent }}$. After Eq. (3) is solved, the utopia target $\boldsymbol{T}^{*}$ that maximizes the utility $V$ is assigned to the engineering problem. The engineering problem then finds an optimal response $\boldsymbol{R}^{L 0}$ to the utopia target with the minimum deviations (Fig. 1).

In most engineering design cases, it is uncommon to meet the utopia target perfectly due to the tradeoff nature of multiple attribute target values or physical feasibility, that is, no feasible design is available to meet the targets perfectly. If the feasible domain in the engineering problem is disconnected in the target space, the engineering design with the minimum deviation from the targets may not correspond to the maximum utility value. Figure 4 shows one-dimensional and two-dimensional cases where the (feasible) minimum deviation from the utopia target does not match the best available utility. Points A and B are both engineering local optima with the minimum deviation from the target. The deviation for point A is smaller, but the corresponding utility is not higher than that of point $B$. Note that these plots are in the target domain instead of the design variable space.

\section{A. Introducing a New Constraint in Enterprise-Level Problem}

The proposed algorithm leads the engineering problem to match the enterprise-driven preferences, that is, targets, by systematically exploring a multiply disconnected feasible design space through the exploration of candidate attribute targets, such that the final design is consistent with the targets and improved in the enterprise utility sense. To enable the move from one feasible domain to another, an additional constraint $C_{\text {aux }}$ is imposed in the enterprise problem [Eq. (3)] based on the achievable engineering response $\boldsymbol{R}^{L 0}$ as shown in Eq. (4), and the enterprise problem is resolved. The physical meaning of the additional constraint is that it imposes a minimum Euclidean distance from the utopia target so that the enterprise problem is forced to find another target for the engineering

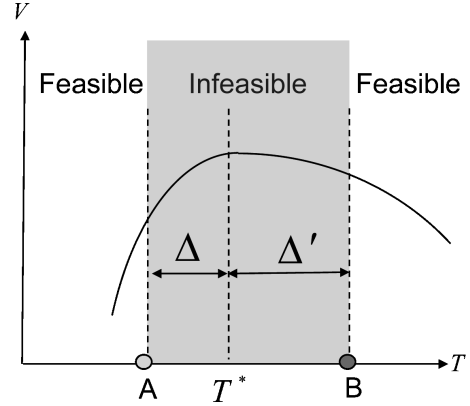

a) One-dimensional case

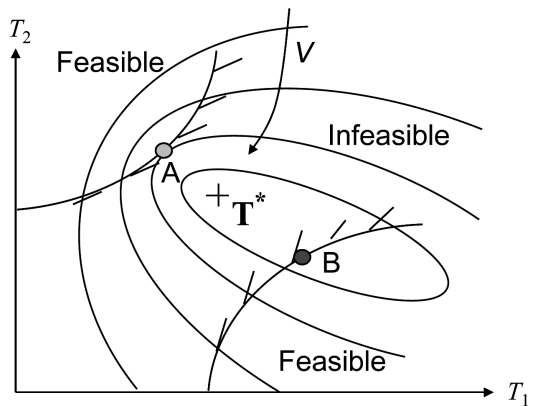

b) Two-dimensional case

Fig. 4 Utilities with engineering feasible domain imposed.

problem. Thus,

$$
C_{\text {aux }}:\left\|\boldsymbol{T}-\boldsymbol{R}^{L 0}\right\| \geq \Delta=\left\|\boldsymbol{T}^{*}-\boldsymbol{R}^{L 0}\right\|
$$

The idea of adding the constraint in Eq. (4) is to explore targets in the new domain that may lead to feasible designs with better enterprise utility. The points inside the circular constraint in Eq. (4) are ruled out because they are infeasible. (Otherwise they should be identified as a solution in the previous iteration because their deviations from the utopia target are less.) For example, in Fig. 4 the engineering problem returns point $\mathrm{A}$ to the enterprise problem with the minimum deviation from the utopia target $\boldsymbol{T}^{*}$. A circular inequality constraint is imposed on the utopia target at the center, with the distance between the utopia target and the engineering response as its radius. The modified enterprise problem $P_{\text {ent }}^{\prime}$ [Eq. (5)] generates a new target $\boldsymbol{T}^{\prime}$ for the engineering problem (Fig. 5). Based on the new target, the engineering problem finds point B as the optimum with the minimum deviation from the new target $\boldsymbol{T}^{\prime}$. Point B is farther from the original utopia target; however, the corresponding utility is higher than that of point A. As a result, point B is selected. The modified enterprise problem is

$$
\begin{aligned}
& P_{\text {ent }}^{\prime}: \max _{T, x_{\text {ent }}} V\left(\boldsymbol{T}, \boldsymbol{x}_{\text {ent }}\right) \\
& \text { subject to } \\
& C_{\text {aux }}:\left\|\boldsymbol{T}-\boldsymbol{R}^{L 0}\right\| \geq \Delta=\left\|\boldsymbol{T}^{*}-\boldsymbol{R}^{L 0}\right\|
\end{aligned}
$$

\section{B. Utilization of Slope Information}

The goal of solving the modified enterprise problem (5) is to lead to another disconnected feasible domain by assigning a new target. However, depending on the slope of the enterprise utility curve, the initial new target for the engineering problem may not sufficiently lead to another disconnected feasible domain; thus, setting the new target may need to be repeated. To visualize this situation, in Fig. 6, a unimodal utility function is plotted with the engineering feasible domain overlapped. The shaded region denotes the infeasible engineering domain, and a disconnected feasible domain exists, on both sides of the infeasible one. At the first iteration the engineering problem returns a response $\left(T^{*}-\Delta\right)$. A constraint, as shown in Eq. (4), is added to the enterprise problem, and the new target is identified at $\left(T^{*}+\Delta\right)$. Take a case when the other feasible domain is farther from the new target $\left(T^{*}+\Delta\right)$ than the previous feasible domain, as 
shown in Fig. 6. The response with the minimum deviation from the target does not change from the previous response $\left(T^{*}-\Delta\right)$, that is, the algorithm returns to the same response as previously found. To avoid terminating the search without exploring farther, additional slope information needs to be utilized to adjust the radius of the restricted feasible domain in the enterprise problem. After finding the minimum deviation engineering design at $\left(T^{*}-\Delta\right)$, the gradient of the utility function, denoted as $\alpha$ is obtained analytically

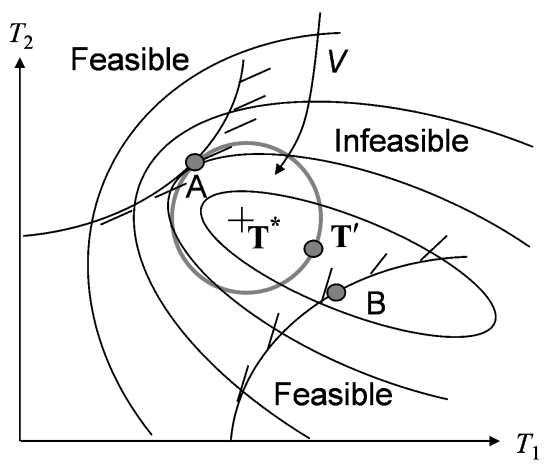

Fig. 5 Circular constraint imposes new target $T^{\prime}$ for the engineering problem.

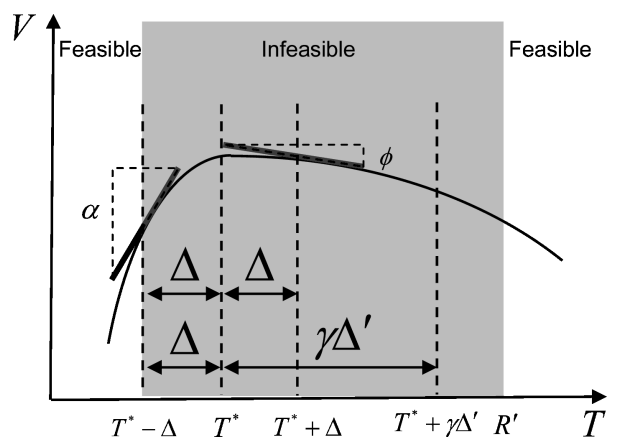

Fig. 6 Updating the radius $\Delta$ in the additional constraint in the modified enterprise problem: slope is considered to set the radius of constraint (4); $\Delta^{\prime}=\|\alpha / \Phi\| \Delta$. or numerically. When the next target for the engineering problem $\left(T^{*}+\Delta\right)$ is obtained by solving the modified enterprise problem, the gradient of the utility function, denoted as $\phi$, is also obtained. If the engineering problem returns the same response $\left(T^{*}-\Delta\right)$ and $|\phi| \leq|\alpha|$, then the constraint radius may be increased up to $\Delta^{\prime}$ with the gradient ratio

$$
\Delta^{\prime}=|\alpha / \phi| \Delta
$$

An updated target $\left(T^{*}+\gamma \Delta^{\prime}\right)$ is assigned to the engineering problem and the engineering problem returns $R^{\prime}$ as response, where $\gamma$ is the step size for updating the new radius of the constraint that takes a value $|\phi / \alpha|<\gamma \leq 1$. When $\gamma=|\phi / \alpha|$, the radius is identical to the original radius $\Delta$, and when $\gamma=1$, the radius becomes $\Delta^{\prime}$ in Eq. (6). As shown in Fig. 7, $\gamma$ can take any value in $|\phi / \alpha|<\gamma \leq 1$ as long as the newly assigned target corresponds to a better utility value. Hence, the enterprise problem repeats assigning a new target by updating $\gamma$ if the engineering problem returns the same response as far as the utility improvement is expected. The the upper limit for increasing the radius of the constraint is provided in Eq. (6), based on a linear approximation of the utility function as shown in Fig. 6.

The proposed iterative procedure is terminated as soon as an engineering level design is found with a better utility, that is, the goal of the solution algorithm is to explore the target space until a feasible engineering design with a better enterprise utility is identified. The proposed algorithm does not attempt to find the global optimum, rather it explores the engineering feasible domain to find alternative feasible design with a better utility if it exists in a disconnected feasible domain. It is also assumed that the engineering product development problem always finds the minimum deviation solution, that is, a feasible design with the minimum deviation from the target. The proposed algorithm is summarized as follows (see also Fig. 7):

1) Start with $\boldsymbol{x}_{0}$.

2) Solve the original enterprise problem $\left(P_{\mathrm{ent}}^{0}\right)$ and find the utopia target $\boldsymbol{T}^{*}$

3) Solve the engineering problem $\left(P_{\text {eng }}\right)$ and obtain the response $\boldsymbol{R}^{L 0}$ with the minimum deviation from the target $\boldsymbol{T}^{*}$.

4) Add an additional constraint $C_{\text {aux }}$ in the enterprise problem, and find a new target $\boldsymbol{T}^{i}$ by solving the modified enterprise problem $\left(P_{\text {ent }}^{\prime}\right)$.

5) Solve the engineering problem and obtain the response $\boldsymbol{R}^{L i}$ with the minimum deviation from the target $\boldsymbol{T}^{i}$.

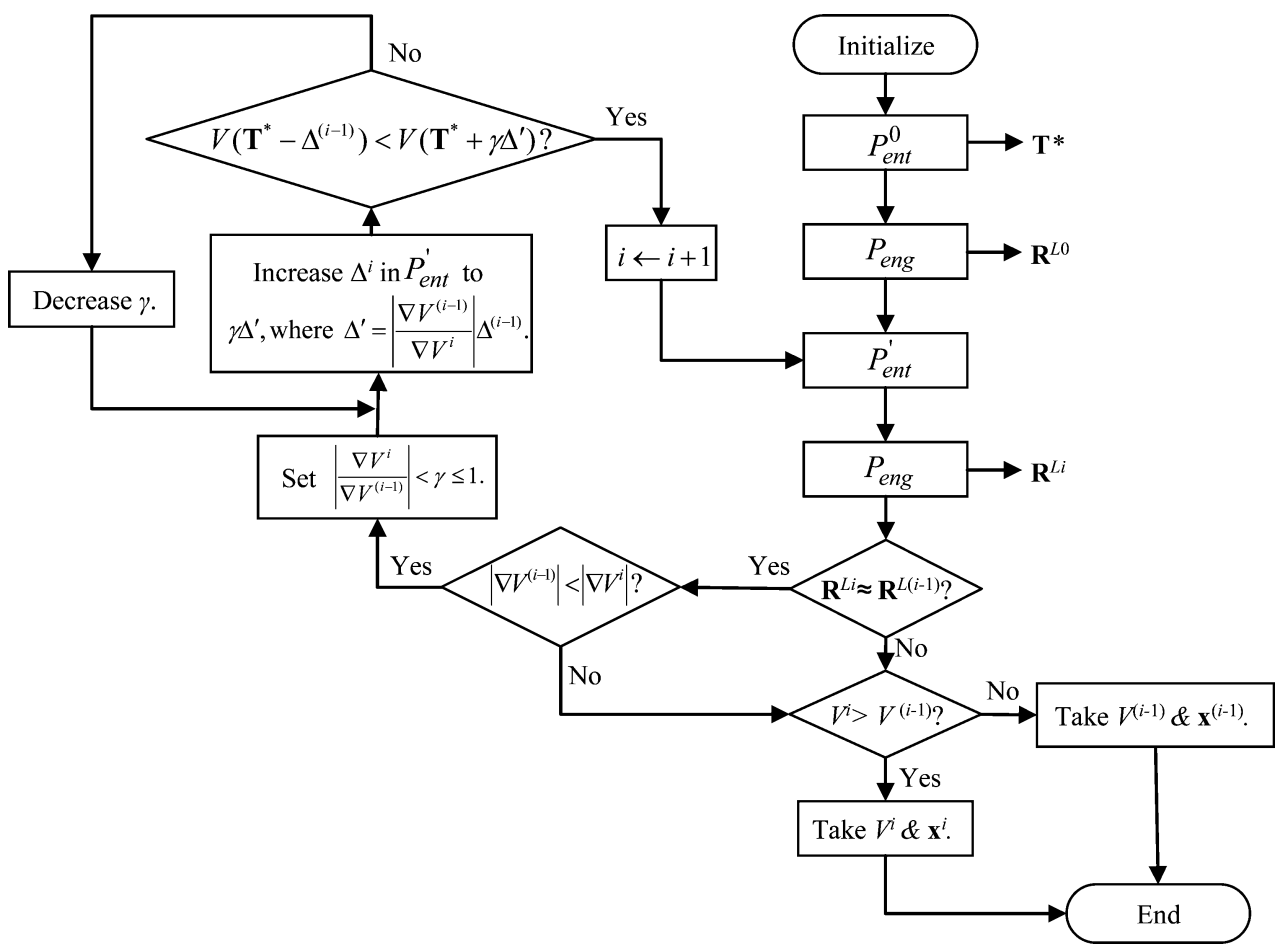

Fig. 7 Solution algorithm. 


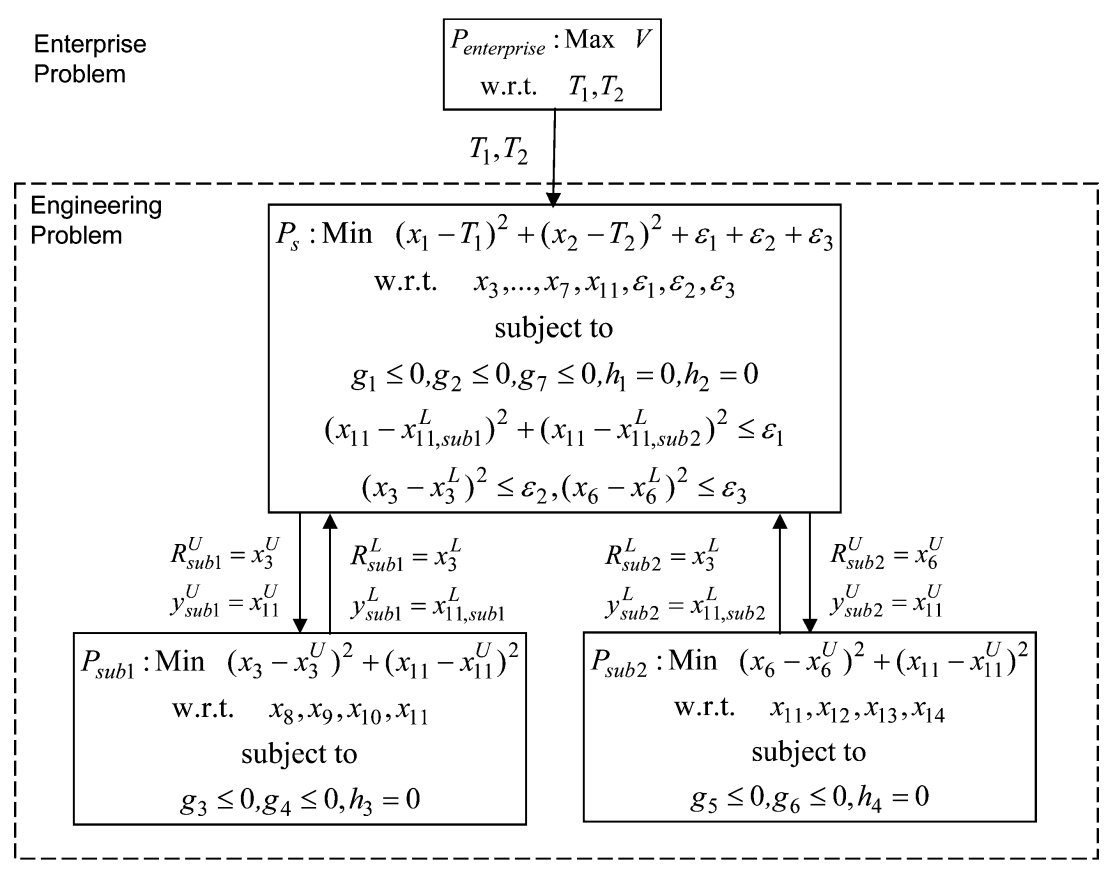

Fig. 8 Decomposed problem hierarchy following analytical target cascading.

6) If $\boldsymbol{R}^{L i} \approx \boldsymbol{R}^{L(i-1)}$ and $\left|\nabla V^{(i-1)}\right|<\left|\nabla V^{i}\right|$, increase $\Delta$ in $C_{\text {aux }}$ in $P_{\text {ent }}^{\prime}$ to $\Delta^{i}=\gamma\left|\nabla V^{(i-1)} / \nabla V^{i}\right| \Delta^{(i-1)}$, where $\left|\nabla V^{i} / \nabla V^{(i-1)}\right| \leq$ $\gamma \leq 1$.

a) If $V\left(T^{*}-\Delta^{(i-1)}\right)<V\left(T^{*}+\Delta^{i}\right)$ go to step 4 and solve $P_{\text {ent }}^{\prime}$

b) Otherwise, decrease $\gamma$ until it satisfies $V\left(T^{*}-\Delta^{(i-1)}\right)<$ $V\left(T^{*}+\Delta^{i}\right)$

7) If $\boldsymbol{R}^{L i} \approx \boldsymbol{R}^{L(i-1)}$ and $\left|\nabla V^{(i-1)}\right| \geq\left|\nabla V^{i}\right|$, compare the current enterprise utility value to the previous one and accept the current design if improved, or accept the previous design.

8) End.

\section{Demonstration and Verifications}

Three examples are presented to demonstrate and verify the elucidated ideas. Two analytical examples show progress to a better enterprise level design while successfully exploring a disconnected feasible engineering domain. The first one has a single utility optimum, and the second has multiple local utility optima at the enterprise level while the engineering feasible domain is disconnected. The lower level problems are decomposed at multiple levels and are solved using ATC. A vehicle design problem involving hierarchical suspension system design is also studied for enterprise profit maximization utilizing real customer purchase data for the demand model along with market share behavior with demographic information.

\section{A. Analytical Examples}

1. Utility with Single Optimum

An analytical geometric programming problem ${ }^{22}$ is modified to demonstrate the proposed algorithm. At the top of the problem hierarchy, the enterprise problem is defined in Eq. (7), and at the bottom level, the engineering problem is defined in Eq. (8). Targets $T_{1}$ and $T_{2}$ are assigned to the engineering level problem [Eq. (8)] after solving the enterprise level problem [Eq. (7)]:

$$
\begin{aligned}
& P_{\mathrm{ent}}^{0}: \min _{T_{1}, T_{2}}-V=\sqrt{\left(T_{1}-2\right)^{2}+\left(4 T_{2}-1\right)^{2}} \\
& +\sqrt{\left(T_{1}-2\right)^{2}+\left(4 T_{2}+1\right)^{2}} \\
& \quad P_{\text {eng }}: \min _{x_{1}, x_{2}, \ldots, x_{14}}\left(x_{1}-T_{1}\right)^{2}+\left(x_{2}-T_{2}\right)^{2}
\end{aligned}
$$

subject to

$$
g_{1}=\left(x_{3}^{-2}+x_{4}^{2}\right) / x_{5}^{2}-1 \leq 0, \quad g_{2}=\left(x_{5}^{2}+x_{6}^{-2}\right) / x_{7}^{2}-1 \leq 0
$$

$$
\begin{gathered}
g_{3}=\left(x_{8}^{2}+x_{9}^{2}\right) / x_{11}^{2}-1 \leq 0, \quad g_{4}=\left(x_{8}^{-2}+x_{10}^{2}\right) / x_{11}^{2}-1 \leq 0 \\
g_{5}=\left(x_{11}^{2}+x_{12}^{-2}\right) / x_{13}^{2}-1 \leq 0, \quad g_{6}=\left(x_{11}^{2}+x_{12}^{2}\right) / x_{14}^{2}-1 \leq 0 \\
g_{7}=-\left[0.1\left(x_{1}-3\right)^{2}+4.5-x_{2}\right]\left[-\left(x_{1}-16\right)^{2}+2-x_{2}\right] \leq 0 \\
h_{1}=x_{1}^{2}-x_{3}^{2}-x_{4}^{-2}-x_{5}^{2}=0, \quad h_{2}=10 x_{2}^{2}-x_{5}^{2}-x_{6}^{2}-x_{7}^{2}=0 \\
h_{3}=x_{3}^{2}-x_{8}^{2}-x_{9}^{-2}-x_{10}^{-2}-x_{11}^{2}=0 \\
h_{4}=x_{6}^{2}-x_{11}^{2}-x_{12}^{2}-x_{13}^{2}-x_{14}^{2}=0 \\
-20 \leq x_{3}, x_{4}, \ldots, x_{14} \leq 20
\end{gathered}
$$

To illustrate hierarchical optimization for engineering product development, the lower level (engineering) problem (8) is decomposed at two levels, system and subsystems (Fig. 8), and solved using the ATC approach. After the top level (enterprise) problem is solved, the targets $T_{1}$ and $T_{2}$ are assigned to the lower level problem $P_{s}$. After solving $P_{s}$, the bottom level problems $P_{\text {sub1 }}$ and $P_{\text {sub2 }}$ are solved based on the targets $x_{3}^{U}, x_{6}^{U}$, and $x_{11}^{U}$ found in that $P_{s}$ problem. Note that $x_{11}$ is a linking variable, that is, common design variable that is

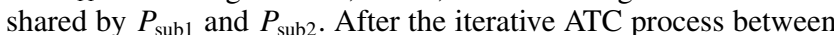
$P_{s}, P_{\text {sub1 } 1}$, and $P_{\text {sub2 }}$ converges, the overall response with respect to the targets $T_{1}$ and $T_{2}$ is passed up to the top (enterprise) level problem and the utility is adjusted completing each iteration in Table 1.

Note that the engineering problem (8) has a disconnected feasible domain with respect to $T_{1}$ and $T_{2}$. This domain is plotted over the utility domain in Fig. 9a. For the unconstrained enterprise level problem, the optimal target, that is, utopia target, is found at $\boldsymbol{T}^{*}=(2.0,0.079)$. Based on the utopia target, the engineering problem finds an optimal response with the minimum deviation at $\boldsymbol{R}^{\text {sys0 }}=(4.006,4.601)$, which is not the best available solution in the enterprise utility sense. With the proposed algorithm, based on the engineering response $\boldsymbol{R}^{\text {sys0}}$, a constraint in Eq. (4) is added to the enterprise level problem and the enterprise level problem is solved again to assign a new target $\boldsymbol{T}^{\prime}$ to the engineering problem. An updated engineering level solution $\boldsymbol{R}^{\text {sys1 }}$ is found near the previous solution $\boldsymbol{R}^{\text {sys0 }}$, that is, the engineering-level response is found in the same feasible domain. The radius for the additional constraint in Eqs. (4) and (5) is increased to $\Delta^{\prime}=\gamma\left|\nabla V^{(i-1)} / \nabla V^{i}\right| \Delta$, where $\left|\nabla V^{i} / \nabla V^{(i-1)}\right| \leq \gamma \leq 1$. A newly assigned target $\boldsymbol{T}^{\prime \prime}$ based on the 
Table 1 Iteration history: utility with single optimum

\begin{tabular}{lcccccc}
\hline \hline Iteration & Target $T_{1}$ & Target $T_{2}$ & Desired utility & Response & Response & Actual utility \\
\hline 1 & 2.000 & 0.079 & -2 & 4.006 & 4.601 & -37.029 \\
2 & 6.478 & -0.005 & -10.093 & 4.772 & 4.814 & -39.855 \\
3 & 9.372 & -0.005 & -15.610 & 15.30 & 1.5 & -29.243 \\
AIO solution & & & & 14.333 & -0.780 & -25.52 \\
\hline \hline
\end{tabular}

Table 2 Iteration history: utility with multiple optima

\begin{tabular}{lcccccc}
\hline \hline Iteration & Target $T_{1}$ & Target $T_{2}$ & Desired utility & Response & Response & Actual utility \\
\hline 1 & 3.837 & 4.695 & 1.903 & 4.259 & 5.216 & -10.17 \\
2 & 2.470 & 3.356 & 1.365 & 2.634 & 3.383 & 0.846 \\
AIO Solution & & & & 2.718 & 3.233 & 0.921 \\
\hline \hline
\end{tabular}

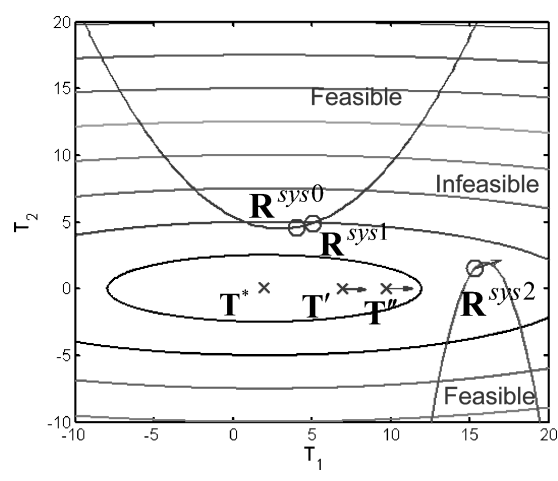

a) Utility with single optimum

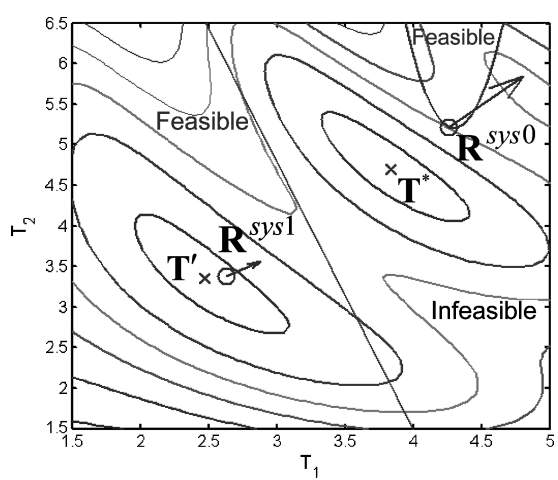

b) Utility with multiple optima

Fig. 9 Disconnected feasible domains mapped over utility space; arrows represent gradient.

slope information [Eq. (6)] from the modified enterprise-level problem guides the engineering-level problem to find the optimal response $\boldsymbol{R}^{\text {sys } 2}$ in the other feasible domain. The corresponding utility value for $\boldsymbol{R}^{\text {sys } 2}$ is better, the lower level response is accepted as a solution, and the algorithm is terminated. At the optimum, the solution is found at $\left(x_{1}, x_{2}, \ldots, x_{14}\right)=(15.30,1.5,5.09,-0.069,0.21$, $4.77,0.30,2.57,0.28,2.55,2.58,-0.84,2.84,2.71)$.

The iteration process is summarized in Table 1 . Note that the responses are getting closer to the AIO solution that is obtained when the decomposed problems in Eqs. (7) and (8) are solved in a single integrated problem.

\section{Utility with Multiple Local Optima}

In this section, a case with multiple local enterprise utility optima is presented. When the utopia utility is not achievable, it is desirable to find a feasible engineering design near the next best local optimal utility. The current example demonstrates that the proposed algorithm enforces the engineering design to move away from the (infeasible) utopia target by imposing the geometric distance constraint. The final design is found around the next best utility rather than the less favorable point near the utopia target. The utility function at the enterprise level with multiple local maxima is defined as follows:

$$
\begin{aligned}
& P_{\text {ent }}^{0}: \\
& \max _{T_{1}, T_{2}} V=2+0.01\left(T_{2}-T_{1}^{2}\right)^{2}+\left(4-T_{1}\right)^{2} \\
& \quad+2\left(4-T_{2}\right)^{2}+7 \sin \left(0.55 T_{1}\right) \sin \left(0.6 T_{1} T_{2}\right)
\end{aligned}
$$

After solving the problem in Eq. (9), targets $T_{1}$ and $T_{2}$ are assigned for the engineering-level design problem

$$
P_{\text {eng }}: \min _{x_{1}, x_{2}, \ldots, x_{14}}\left(x_{1}-T_{1}\right)^{2}+\left(x_{2}-T_{2}\right)^{2}
$$

subject to

$$
\begin{gathered}
g_{1}=\left(x_{3}^{-2}+x_{4}^{2}\right) / x_{5}^{2}-1 \leq 0, \quad g_{2}=\left(x_{5}^{2}+x_{6}^{-2}\right) / x_{7}^{2}-1 \leq 0 \\
g_{3}=\left(x_{8}^{2}+x_{9}^{2}\right) / x_{11}^{2}-1 \leq 0, \quad g_{4}=\left(x_{8}^{-2}+x_{10}^{2}\right) / x_{11}^{2}-1 \leq 0 \\
g_{5}=\left(x_{11}^{2}+x_{12}^{-2}\right) / x_{13}^{2}-1 \leq 0, \quad g_{6}=\left(x_{11}^{2}+x_{12}^{2}\right) / x_{14}^{2}-1 \leq 0 \\
g_{7}=-\left(10\left(x_{1}-4.3\right)^{2}+5.2-x_{2}\right)\left(-3.3\left(x_{1}-4.15\right)+1-x_{2}\right) \leq 0 \\
h_{1}=x_{1}^{2}-x_{3}^{2}-x_{4}^{-2}-x_{5}^{2}=0, \quad h_{2}=x_{2}^{2}-x_{5}^{2}-x_{6}^{2}-x_{7}^{2}=0 \\
h_{3}=x_{3}^{2}-x_{8}^{2}-x_{9}^{-2}-x_{10}^{-2}-x_{11}^{2}=0 \\
h_{4}=x_{6}^{2}-x_{11}^{2}-x_{12}^{2}-x_{13}^{2}-x_{14}^{2}=0 \\
-20 \leq x_{3}, x_{4}, \ldots, x_{14} \leq 20
\end{gathered}
$$

The engineering problem is decomposed and solved as in the first example. The contour plot of the utility function with the overlapped engineering-level constraints $g_{7}$ is presented in Fig. 9b. For the unconstrained enterprise-level problem, the optimal (utopia) target is found at $\boldsymbol{T}^{*}=(3.837,4.695)$. Based on this target, the engineeringlevel problem finds an optimal response with the minimum deviation at $\boldsymbol{R}^{\text {sys0 }}=(4.259,5.216)$. Based on response $\boldsymbol{R}^{\text {sys } 0}$, a constraint, as shown in Eq. (4), is added to the enterprise-level problem and the enterprise-level problem is solved again to assign a new target $\boldsymbol{T}^{\prime}$ to the engineering-level problem. The additional constraint guides the search process to find another local optimum in the utility space and assigns a new target for the engineering-level problem. The engineering-level problems find an optimal solution $\boldsymbol{R}^{\text {sys1 }}$ in the other region of the feasible domain. After checking that the corresponding utility value for $\boldsymbol{R}^{\text {sys1 }}$ is better than the previous one, the engineering-level response is accepted as a solution. At the optimum, the solution is found at $\left(x_{1}, x_{2}, \ldots, x_{14}\right)=(2.63,3.38,2.19$, $0.94,1.05,3.03,1.10,0.92,-1.13,-0.96,1.45,0.85,1.87,1.68)$. The iteration process is summarized in Table 2.

The two analytical examples demonstrate that the proposed approach successfully explores the enterprise utility space to meet the enterprise objective with a consistent feasible engineering design. Note that the final solutions in Tables 1 and 2 have deviations from the reference AIO solution, mainly contributed by the way target values are set. The AIO reference solution is based on the utopia target, and the current solution is based on the updated new targets, satisfying the geometric constraint. In other words, the focus of the proposed algorithm is not on matching the AIO solution exactly, 


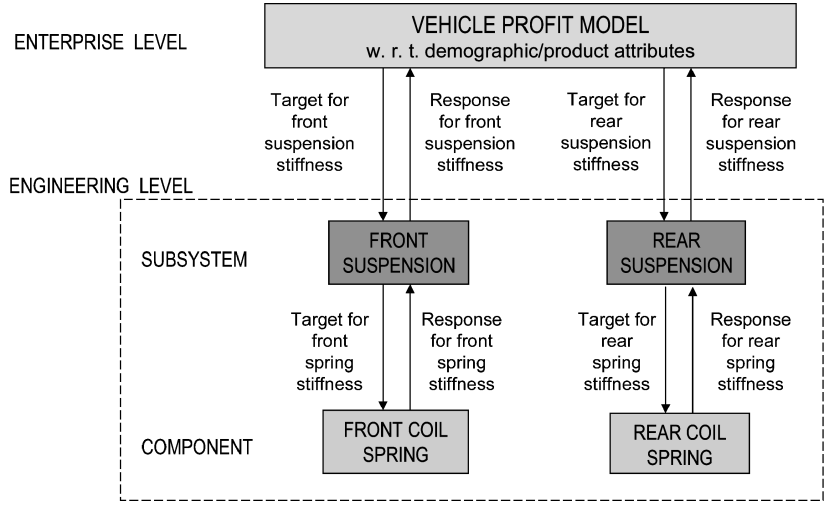

Fig. 10 Schematic of vehicle profit and suspension design model.

rather it focuses on a systematic exploration of the feasible space to generate the final solution near the AIO solution. Additionally, in case there exists no additional disconnected feasible domain, the algorithm can still find the best feasible design.

\section{B. Enterprise-Driven Multilevel Vehicle Suspension Design}

The algorithm is now applied to an enterprise-driven vehicle design problem with emphasis on vehicle suspension design at the product development level. As shown in Fig. 9, at the enterprise level, the vehicle profit model is created based the customers' demographic attributes and all critical vehicle system attributes. Because the product development level focuses only on the suspension for vehicle ride quality improvement, only the targets for front and rear suspension stiffness parameters are treated as variables at the enterprise level. Modeling details of the vehicle chassis composed of front/rear double A-arm suspension and vertical/cornering tire models can be found by Kim et al. ${ }^{24}$ ATC successfully simulated the vehicle chassis design process based on the targets for handling and ride quality of a sport-utility vehicle. Here a simplified chassis system composed of front/rear suspension subsystems and coil spring components is considered for the engineering product development task (Fig. 10). Some details of creating the demand and profit models are provided first.

\section{Medium-Size Vehicle Demand Model}

At the enterprise level, a demand model is created to analyze the effect of incremental engineering design changes on the market share of the vehicle ${ }^{24}$ using the DCA method. ${ }^{9}$ A key concept in DCA is the use of random utility to address unobserved taste variations, unobserved attributes, and model deficiencies. Random utility entails the assumption that the individual customer's true utility $U_{\text {in }}$ consists of a deterministic or observable part $W_{\text {in }}$ and a random unobservable disturbance $\varepsilon_{\text {in }}$ :

$$
U_{\text {in }}=W_{\text {in }}+\varepsilon_{\text {in }}
$$

A quantitative process based on multinomial analysis is used to generate the demand model. The deterministic part of the utility can be parameterized as a function of observable independent variables (customer-product-selection attributes $\boldsymbol{A}$, engineering attributes $\boldsymbol{E}$, socioeconomic and demographic attributes $S$, and price $P$ ) and unknown coefficients $\beta$, which can be estimated by observing the choices respondents make and, thus, represent the respondents' preference. The $\beta$ coefficients and utility functions are indicated with the subscript $n$, representing the $n$th respondent, whereas the index $i$ refers to the $i$ th choice alternative. There is no functional form imposed on the utility function $W$. For the purpose of the current study, an additive form of the utility function, linear in the $\beta$ coefficients is used. Thus,

$$
W_{\text {in }}=f\left(\boldsymbol{A}_{i}, \boldsymbol{E}_{i}, P_{i}, \boldsymbol{S}_{n}: \beta_{n}\right)
$$

Based on the market data from J.D. Power and Associates and the vehicle attribute descriptions from Ward's automotive yearbook, ${ }^{35}$ the demand model $Q$ is created as a function of demographic and product attributes such as income; age; retail price; resale value;

\begin{tabular}{|c|c|c|c|}
\hline Description & $\beta$ Coefficient & $t$ Value & $\begin{array}{l}\text { 95\% Confidence } \\
\text { interval }\end{array}$ \\
\hline \multicolumn{4}{|c|}{ Demographic variable } \\
\hline Income 2 & 0.13 & 6.41 & $(0.09,0.18)$ \\
\hline Income 3 & 0.01 & 0.48 & $(-0.03,0.05)$ \\
\hline Income 4 & 0.06 & 2.57 & $(0.01,0.11)$ \\
\hline Income 5 & -0.10 & -4.2 & $(-0.15,-0.05)$ \\
\hline Income 6 & -0.08 & -3.37 & $(-0.13,-0.03)$ \\
\hline Income 7 & 0.07 & 2.99 & $(0.02,0.11)$ \\
\hline Income 8 & 0.08 & 3.22 & $(0.03,0.12)$ \\
\hline Income 9 & 0.08 & 3.25 & $(0.03,0.14)$ \\
\hline Income 10 & 0.19 & 9.38 & $(0.15,0.23)$ \\
\hline Income 11 & 0.05 & 2.29 & $(0.01,0.10)$ \\
\hline Income 12 & 0.04 & 1.18 & $(-0.02,0.10)$ \\
\hline Age & 0.13 & 12.37 & $(0.11,0.15)$ \\
\hline \multicolumn{4}{|c|}{ Product variable } \\
\hline Retail price & -1.57 & -4.14 & $(-2.31,-0.82)$ \\
\hline Resale value & 2.15 & 2.54 & $(0.49,3.80)$ \\
\hline VDI & -1.69 & -1.49 & $(-3.92,0.53)$ \\
\hline APR & -1.05 & -1.34 & $(-2.58,0.49)$ \\
\hline Product fuel economy & 0.64 & 1.51 & $(-0.19,1.46)$ \\
\hline Vehicle length & -0.60 & -0.5 & $(-2.95,1.74)$ \\
\hline Front suspension stiffness & 1.75 & 3.11 & $(0.65,2.85)$ \\
\hline Rear suspension stiffness & 0.88 & 1.28 & $(-0.47,2.24)$ \\
\hline
\end{tabular}

Table 3 Results of demand model estimation

vehicle dependability index (VDI), a quality measure, expressd in terms of defects per 100 parts; annual percentage rate (APR) of loan; fuel economy; vehicle length; front suspension stiffness; and rear suspension stiffness (Table 3). The modle makes prediction on the change in market share, that is, change in the number of vehicles sold of a particular make. In the demand model representing the midsize car segment, 12 vehicles ( 7 models and 12 trim levels) are considered. Considering other segments, for example, sports models or pickup trucks, would run the risk of yielding models that have a heavy demographic bias and therefore are not very sensitive to changes in product attributes. The assumption is that customers only consider vehicles from the midsize car segment, and specifically the 12 vehicle trims, when making their decision. Note that the demographic attributes can only be included as alternative specific variables ${ }^{9}$ due to the nature of the MNL. In the current example, there are 12 vehicle alternatives, considering the various midsize car trims. If a demographic attribute, such as age or income of the respondent, should be included in the model, the coefficients for a maximum 11 variables can be estimated for each demographic attribute. Each of these 11 variables corresponds to one of the alternatives or car trims. The demographic variable corresponding to at least one of the alternatives, for example, alternative 1, has to be fixed. Usually, the coefficient for the alternative that is fixed is set to be zero. The coefficients for the rest of the alternatives, alternatives 2-12, are then estimated with respect to the reference alternative 1 . In the demand model used here, 11 income variables corresponding to alternatives 2-12 are estimated, and they are evaluated with alternative 1 as reference. Similarly, the age variable is assumed to be equal for all domestic cars and is evaluated with respect to imported cars. The number of survey correspondents was 3881 . Front and rear suspension spring stiffnesses are used to model suspension characteristics.

Starting with a baseline specification that includes alternative specific constants, that is, essential product attributes and demographic variables, the demand model is improved incrementally by adding additional attributes to the model. Alternative specific constants, such as fuel economy and suspension stiffnesses, are added to represent the average preference of individuals for an alternative relative to a reference alternative and also to account for the average effect of all explanatory variables. The estimated models are then evaluated on several criteria including behavioral realism or their ability to model customer behavior in line with the analyst's expectations and goodness of empirical fit to the data. The model estimation is carried out by maximizing the log likelihood using STATA. ${ }^{36}$

Table 3 includes results of the model estimation. Several observations can be made. Negative signs of retail price, VDI, APR, and 
vehicle length mean that customers prefer lower values for these variables, that is, customers prefer cheaper cars, lower interest rates, fewer defects, and cars that facilitate easy parking. Positive sign for fuel economy means that customers prefer higher gas mileage and positive signs for the suspension stiffnesses mean that stiffer suspensions are preferred. A stiffer suspension generally translates to better handling and load-carrying abilities but also results in a harsher ride. In this context, the current choice model indicates that customers value handling characteristics more than ride quality. Also, because we are dealing with variables normalized with respect to their extreme values, the magnitude of the coefficients should reflect their relative importance.

Enterprise level utility is defined as the change of total profit [Eq. (13)], which leads to Eq. (14), a function of demand and cost. Price of a vehicle $P$ is assumed as a constant, and $C_{\text {susp }}$ and $C_{0}$ represent costs for the suspension subsystems and for the rest of the vehicle system, respectively,

$$
\Pi=Q(\boldsymbol{A}, \boldsymbol{E}, \boldsymbol{S}) \times\left(P-C_{\text {susp }}-C_{0}\right)
$$

Suspension cost is assumed to be linearly proportional to suspension stiffnesses and vehicle price. Among the customer-driven attributes in the demand model, only front/rear suspension stiffnesses are considered as variables for target setting, whereas the rest of the attributes are set constant at the baseline values [Eq. (14)]. In the current study values for a domestic sedan with $P=20,000 \$, C_{0}=18,100 \$, a_{f}=0.05 \mathrm{kN} / \mathrm{m}^{-1}, a_{r}=0.05 \mathrm{kN} / \mathrm{m}^{-1}$, $k_{s f 0}=25.5 \mathrm{kN} / \mathrm{m}$, and $k_{s r 0}=19.5 \mathrm{kN} / \mathrm{m}$ are used:

$$
\begin{aligned}
\Delta \Pi= & Q\left(k_{s f}, k_{s r}\right) \times\left[\left(1-a_{f} k_{s f}-a_{r} k_{s r}\right) P-C_{0}\right] \\
& -Q\left(k_{s f 0}, k_{s r 0}\right) \times\left[\left(1-a_{f} k_{s f 0}-a_{r} k_{s r 0}\right) P-C_{0}\right]
\end{aligned}
$$

Current demand model indicates that softer front suspension and stiffer rear suspension lead to the most profit for the market (Fig. 11). In Fig. 11, the shaded regions in the plot of change of profit vs the suspension design targets indicate a disconnected feasible domain for the suspension design.

\section{Implementation of Proposed Algorithm}

In the preceding vehicle chassis design example by ATC, ${ }^{24}$ the targets for the chassis system design are given based on experience, that is, fixed target values. In the current formulation, the targets are set based on maximizing the enterprise utility, that is, profit of a firm. Based on the targets, ATC cascades top-level design targets to

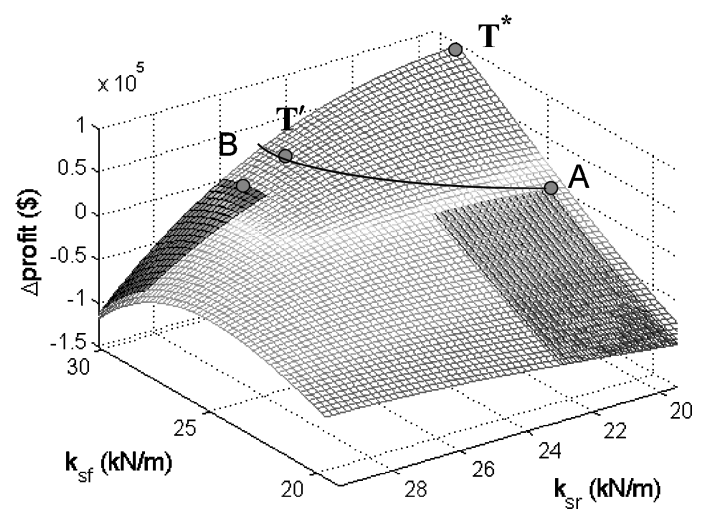

Fig. 11 Vehicle profit model: profit change with respect to suspension stiffness changes; solid curve connecting $A$ and $T^{\prime}$ indicates geometric distance constraint. system, subsystem, and components. In the current example, only front and rear vehicle suspension subsystems and spring components are included in the hierarchy for simplicity.

Table 4 summarizes the iteration process for the algorithm. The utopia target for suspension design is given at $\boldsymbol{T}^{*}=(30.2,19.5) \mathrm{kN} / \mathrm{m}$ with profit $\$ 80,460$ corresponding to the peak point on the utility surface in Fig. 11. Because of engineering feasibility, the design with the minimum deviation from the utopia target is found at point $\boldsymbol{A}=(25.0,19.5) \mathrm{kN} / \mathrm{m}$ with $-\$ 8935$ profit, that is, loss. Based on this design, the algorithm imposes a limiting constraint [Eq. (4)] to the enterprise problem. After adding the constraint in Fig. 11 (solid curve), the modified enterprise problem finds a new optimal target with the maximum utility at $\boldsymbol{T}^{\prime}=(28.6,24.5) \mathrm{kN} / \mathrm{m}$ with $\$ 33,701$ profit. This new target guides the ATC process to reach design $\boldsymbol{B}=(29.4,25.0) \mathrm{kN} / \mathrm{m}$ with the improved profit $\$ 27,234$. Note that the utopia targets assigned to the ATC problem are not achievable due to engineering feasibility. However, the algorithm guides the iteration process to a design where the best available utility is obtained. Detailed suspension and coil spring designs by ATC are summarized in Tables 5-8.

Table 5 Front suspension design

\begin{tabular}{lccc}
\hline \hline Rear suspension subsystem design & $\begin{array}{c}\text { Optimal } \\
\text { value }\end{array}$ & $\begin{array}{c}\text { Lower } \\
\text { bound }\end{array}$ & $\begin{array}{c}\text { Upper } \\
\text { bound }\end{array}$ \\
\hline Linear coil spring stiffness, N/mm & 113.4 & 30 & 160 \\
Spring free length, mm & 375.1 & 300 & 650 \\
Torsional stiffness, N.m/deg & 30 & 20 & 85 \\
Overall rear suspension stiffness, N/mm & 29.3 & 19 & 30.2 \\
Suspension travel, m & 0.099 & 0.05 & 0.1 \\
\hline \hline
\end{tabular}

Table 6 Rear suspension design

\begin{tabular}{lccc}
\hline \hline Rear suspension subsystem design & $\begin{array}{c}\text { Optimal } \\
\text { value }\end{array}$ & $\begin{array}{c}\text { Lower } \\
\text { bound }\end{array}$ & $\begin{array}{c}\text { Upper } \\
\text { bound }\end{array}$ \\
\hline Linear coil spring stiffness, N/mm & 70.5 & 30 & 160 \\
Spring free length, mm & 472.4 & 300 & 650 \\
Torsional stiffness, N.m/deg & 69.3 & 20 & 85 \\
Overall rear suspension stiffness, N/mm & 25 & 19 & 30.2 \\
Suspension travel, m & 0.099 & 0.05 & 0.1 \\
\hline \hline
\end{tabular}

Table 7 Front coil spring design

\begin{tabular}{lccc}
\hline \hline Front coil spring component design & $\begin{array}{c}\text { Optimal } \\
\text { value }\end{array}$ & $\begin{array}{c}\text { Lower } \\
\text { bound }\end{array}$ & $\begin{array}{c}\text { Upper } \\
\text { bound }\end{array}$ \\
\hline Wire diameter, $\mathrm{m}$ & 0.015 & 0.005 & 0.03 \\
Coil diameter, $\mathrm{m}$ & 0.077 & 0.05 & 0.2 \\
Pitch & 0.04 & 0.04 & 0.1 \\
Linear coil spring stiffness, N/mm & 114.3 & & \\
Spring bending stiffness, $\mathrm{N} \cdot \mathrm{m} / \mathrm{deg}$ & 28.5 & & \\
\hline \hline
\end{tabular}

Table 8 Rear coil spring design

\begin{tabular}{lccc}
\hline \hline Rear coil spring component design & $\begin{array}{c}\text { Optimal } \\
\text { value }\end{array}$ & $\begin{array}{c}\text { Lower } \\
\text { bound }\end{array}$ & $\begin{array}{c}\text { Upper } \\
\text { bound }\end{array}$ \\
\hline Wire diameter, $\mathrm{m}$ & 0.02 & 0.005 & 0.03 \\
Coil diameter, $\mathrm{m}$ & 0.154 & 0.05 & 0.2 \\
Pitch & 0.05 & 0.04 & 0.1 \\
Linear coil spring stiffness, N/mm & 73.4 & & \\
Spring bending stiffness, N.m/deg & 59.3 & & \\
\hline \hline
\end{tabular}

Table 4 Iteration history: maximizing profit with vehicle suspension design change

\begin{tabular}{lccccrr}
\hline \hline Iteration & $\begin{array}{c}\text { Target } \\
k_{s f}, \mathrm{kN} / \mathrm{m}\end{array}$ & $\begin{array}{c}\text { Target } \\
k_{s r}, \mathrm{kN} / \mathrm{m}\end{array}$ & $\begin{array}{c}\text { Desired } \\
\text { utility, } \$\end{array}$ & $\begin{array}{c}\text { Response } \\
k_{s f}, \mathrm{kN} / \mathrm{m}\end{array}$ & $\begin{array}{c}\text { Response } \\
k_{s r}, \mathrm{kN} / \mathrm{m}\end{array}$ & $\begin{array}{c}\text { Actual } \\
\text { utility, \$ }\end{array}$ \\
\hline 1 & 30.2 & 19.5 & 80460 & 25.0 & 19.5 & -8935 \\
2 & 28.6 & 24.5 & 33701 & 29.4 & 25.0 & 27234 \\
\hline \hline
\end{tabular}




\section{Conclusions}

In this work, enterprise product planning has been systematically linked to the hierarchical product development process under the multilevel optimization framework. To ensure preference consistency when solving decomposed enterprise and engineering problems, an algorithm was developed to explore systematically the product attribute targets set at the enterprise level to lead the engineering product development to feasible and optimal designs in the enterprise context. To explore the disconnected feasible domain in the target space, an additional set of constraints was added in the enterprise-level problem by imposing a Euclidean distance from the utopia unachievable target, forcing exploration of new target values at the enterprise level. For robust exploration, additional sensitivity information with respect to the enterprise utility, that is, gradient of the utility function, was utilized when imposing the Euclidean distance constraint from the utopia target. If the utility was less sensitive to the changes of targets, a bigger geometric distance constraint was imposed to explore the target space further. This sensitivity information was used only in the enterprise-level problem and not passed down to the engineeringlevel problem. Only targets and responses were passed between the enterprise and engineering problems. Example problems successfully demonstrated the proposed approach by exploring disconnected feasible engineering design space. Although it is not common to achieve aggressive product performance targets perfectly, systematically assigning targets to the engineering problem can lead to a better product design with better enterprise level utility. From the two mathematical examples, we note that the proposed algorithm works effectively for problems with disconnected target space; it also has the capability to escape infeasible local utility optima.

The vehicle design example captures the disjoint nature of the product performance targets and manufacturing limitations. With the current algorithm, the final optimal design is a better design in maximizing the profit of a firm, as well as meeting the feasibility requirements imposed in the vehicle suspension design specifications. MNL model was successfully incorporated in developing the demand model that was part of the enterprise-level objective for the vehicle case study. Also ATC was successfully incorporated to achieve the performance targets in the hierarchical engineering product development process.

The proposed algorithm is applicable to a general multilevel multidisciplinary design case where the lower level performance, that is, specification, space is imposed a disconnected feasible domain at the higher level. Note that if all design variables are continuous with the exception of the target space, traditional optimization algorithms such as sequential quadratic programming (SQP) can still be applied in both enterprise- and engineering-level problems. The results of the two analytical examples in Sec. IV were obtained by SQP in MATLAB ${ }^{\circledR}$.

The number of disconnected feasible domains in the examples was two; however, the method is not limited to just two-domain cases. After updating the Euclidean distance radius in the additional constraint at the enterprise level, a global search algorithm can be incorporated at the engineering design level. Currently, the algorithm terminates as soon as it finds a better utility or confirms that the current utility is the best after increasing the radius to the upper limit. However, this algorithm can be applied repeatedly in combination with global search to handle the case where the utility function at the enterprise level has multiple optima. Future work will involve introducing uncertainty in multilevel optimization and adopting the nested MNL model for demand modeling, which can be more effectively incorporated in multilevel decision making scenarios, as well as in heterogeneous market segments, for example, considering sedans with sport utility vehicles. Furthermore, the sensitivity of profit to multiple product attributes from the enterprise model can be utilized to refine the objective in the engineering product development model.

\section{Acknowledgments}

This work has been supported at Northwestern University by National Science Foundation (NSF) Grant DMII 0335880 and the Ford University Research Program, the U.S. Army Tank-Automotive Research, Development and Engineering Center through the Automotive Research Center at the University of Michigan and the Dual Use Science and Technology Program, and a collaborative NSF research grant between Northwestern University (DMI-0503781) and the University of Michigan (DMI-0503737). J.D. Power and Associates is also acknowledged for providing vehicle-quality survey data. The authors appreciate helpful suggestions by Frank Koppelman and Henk Jan Wassenaar at Northwestern University and Geoff Rideout at University of Michigan.

\section{References}

${ }^{1}$ Donndelinger, J., and Cook, H., "Methods for Analyzing the Value of Vehicles," Society of Automotive Engineers, SAE Paper 970762, Feb. 1997.

${ }^{2}$ Besharati, B., Azarm, S., and Farhang-Mehr, A., "A Customer-Based Expected Utility Metric for Product Design Selection,' Proceedings of DETC 2002 ASME Design Engineering Technical Conference, DETC2002/DAC34081, American Society of Mechanical Engineers, 2002.

${ }^{3} \mathrm{Li}, \mathrm{H}$., and Azarm, S., "Product Design Selection Under Uncertainty and with Competitive Advantage," Proceedings of DETC 2000 ASME Design Engineering Technical Conference, DETC2000/DAC-14234, American Society of Mechanical Engineers, 2000.

${ }^{4}$ Wassenaar, H., and Chen, W., "An Approach to Decision Based Design with Discrete Choice Analysis for Demand Modeling," Journal of Mechanical Design, Vol. 125, No. 3, 2003, pp. 490-497.

${ }^{5}$ Cooper, A. B., Georgiopoulos, P., Kim, H. M., and Papalambros, P. Y., "Analytical Target Setting: An Enterprise Context in Optimal Product Design," Journal of Mechanical Design (to be published); also American Society of Mechanical Engineers, Design Automation Conf., Paper DAC-48734, Sept. 2003.

${ }^{6} \mathrm{Gu}$, X., Renaud, J., Ashe, L., Batill, S., Budhiraja, A., and Krajewski, L., "Decision-Based Collaborative Optimization," Journal of Mechanical Design, Vol. 124, No. 1, 2002, pp. 1-13.

${ }^{7}$ Michalek, J., Feinberg, F., and Papalambros, P., "Linking Marketing and Engineering Product Design Decisions via Analytical Target Cascading," Journal of Product Innovation Management: Special Issue on Design and Marketing in New Product Development, Vol. 22, 2005, pp. 42-62.

${ }^{8}$ Markish, J., and Willcox, K., "Value-Based Multidisciplinary Techniques for Commercial Aircraft System Design," AIAA Journal, Vol. 41, No. 10, 2003, pp. 2004-2012.

${ }^{9}$ Ben-Akiva, M., and Lerman, S., Discrete Choice Analysis, MIT Press, Cambridge, MA, 1985.

${ }^{10}$ Hazelrigg, G., Systems Engineering: An Approach to InformationBased Design, Prentice-Hall, Upper Saddle River, NJ, 1996.

${ }^{11}$ Wassenaar, H. J., Chen, W., Cheng, J., and Sudjianto, A., "Enhancing Discrete Choice Demand Modeling for Decision-Based Design," Journal of Mechanical Design, Vol. 124, No. 4, 2005, pp. 514-523.

${ }^{12}$ Wassenaar, H. J., Chen, W., Cheng, J., and Sudjianto, A., "An Integrated Latent Variable Choice Modeling Approach to Enhancing Product Demand Modeling," Proceedings of DETC 2004 ASME Design Engineering Technical Conference, DETC2004-57487, American Society of Mechanical Engineers, 2004

${ }^{13}$ Badhrinath, K., and Rao, J. R. J., "Modeling for Concurrent Design Using Game Theory Formulations," Concurrent Engineering: Research and Applications, Vol. 4, No. 4, 1996, pp. 389-399.

${ }^{14}$ Rao, J. R. J., and Chidambaram, B., "Parametric Deformations and Model Optimality in Concurrent Design," Advances in Design Automation, DE-Vol. 65-2, No. 2, 1993, pp. 477-485.

${ }^{15}$ Pakala, R., and Rao, J. R. J., "A Study of Concurrent DecisionMaking Protocols in the Design of a Metal Cutting Tool Using Monotonicity Arguments," Engineering Optimization, Vol. 27, No. 3, 1996, pp. 229-264.

${ }^{16}$ Lewis, K., and Mistree, F., "Modeling Interactions in Multidisciplinary Design: A Game Theoretic Approach," AIAA Journal, Vol. 35, No. 8, 1997, pp. 1387-1392.

${ }^{17}$ Rao, J. R. J., Badhrinath, K., Pakala, R., and Mistree, F., "A Study of Optimal Design Under Conflict Using Models of Multi-Player Games," Engineering Optimization, Vol. 28, No. 1-2, 1997, pp. 63-94.

${ }^{18}$ Balas, E., "Disjunctive Programming," Annals of Discrete Mathematics, Vol. 5, 1979, pp. 3-51.

${ }^{19}$ Turkay, M., and Grossmann, I., "Disjunctive Programming Techniques for the Optimization of Process Systems with Discontinuous Investment 
Costs-Multiple Size Regions," Industrial and Engineering Chemistry Research, Vol. 35, No. 8, 1996, pp. 2611-2623.

${ }^{20}$ Owen, J., "Disjunctive Approaches for Solving General Mixed-Integer Linear Programs," Ph.D. Dissertation, Dept. of Industrial Engineering and Management Sciences, Northwestern Univ., Evanston, IL, 1998.

${ }^{21}$ Michelena, N., Kim, H. M., and Papalambros, P. Y., "A System Partitioning and Optimization Approach to Target Cascading," Proceedings of the 12th International Conference on Engineering Design, Vol. 24, The Design Society, Glasgow, Scotland, U.K., 1999, pp. 1109-1112.

${ }^{22}$ Kim, H. M., "Target Cascading in Optimal System Design," Ph.D. Dissertation, Dept. of Mechanical Engineering, Univ. of Michigan, Ann Arbor, MI, 2001.

${ }^{23}$ Kim, H. M., Michelena, N. F., and Papalambros, P. Y., "Target Cascading in Optimal System Design," Journal of Mechanical Design, Vol. 125, No. 3, 2003, pp. 474-480.

${ }^{24}$ Kim, H. M., Rideout, D. G., Papalambros, P. Y., and Stein, J. L., "Analytical Target Cascading in Automotive Vehicle Design," Journal of Mechanical Design, Vol. 125, No. 3, 2003, pp. 481-489.

${ }^{25}$ Sobieszczanski-Sobieski, J., "Optimization by Decomposition: A Step from Hierarchic to Nonhierarchic Systems," NASA CP-3031, NASA Langley Research Center, Hampton, VA, 1988.

${ }^{26}$ Sobieszczanski-Sobieski, J., Agte, J., and Sandusky, R., "Bi-Level Integrated System Synthesis (BLISS)," NASA TM-1998-208715, NASA Langley Research Center, Hampton, VA, 1998.

${ }^{27}$ Braun, R., "Collaborative Optimization: An Architecture for LargeScale Distributed Design," Ph.D. Dissertation, Dept. of Aeronautics and Astronautics, Stanford Univ., Stanford, CA, 1996.

${ }^{28}$ Sobieszczanski-Sobieski, J., and Haftka, R., "Multidisciplinary Aerospace Design Optimization Survey of Recent Developments," Struc- tural Optimization, Vol. 14, No. 1, 1997, pp. 1-23.

${ }^{29}$ Kroo, I., "Multidisciplinary Optimization Applications in Preliminary Design—Status and Directions," AIAA Paper 97-1408, Sept. 1997.

${ }^{30}$ Green, P., and Srinivasan, V., "Conjoint Analysis Marketing: New Developments with Implications Research and Practice," Journal of Marketing, Vol. 54, Oct. 1990, pp. 3-19.

${ }^{31}$ Koppelman, F., and Sethi, V., Handbook of Transport Modeling, Pergamon, Oxford, 2000.

${ }^{32}$ Michalek, J. J., and Papalambros, P. Y., "An Efficient Weighting Update Method to Achieve Acceptable Consistency Deviation in Analytical Target Cascading," Journal of Mechanical Design, Vol. 127, No. 2, 2005, pp. 206-214.

${ }^{33}$ Kim, H. M., Chen, W., and Wiecek, M., "Lagrangian Dual Coordination for Analytical Target Cascading," AIAA Journal (submitted for publication); also International Federation of Operational Research Societies Conf., July 2005.

${ }^{34}$ Tosserams, S., Etman, L. F. P., Papalambros, P. Y., and Rooda, J. E., "An Augmented Lagrangian Realization for Analytical Target Cascading using the Alternating Directions Method of Multipliers," 6th World Congress of Structural and Multidisciplinary Optimization, International Society for Structural and Multidisciplinary Optimization, 2005.

${ }^{35}$ Ward's Automotive Yearbook, Ward's Communications, Southfield, MI, 2004.

${ }^{36}$ Stata Programming Reference Manual, STATA Corp., College Station, TX, 2003.

A. Messac Associate Editor 